\title{
ANORMALIDADES ANATÔMICAS DAS VIAS RESPIRATÓRIAS DO BRAQUICÉFALO E SUAS PRINCIPAIS TÉCNICAS DE CORREÇÃO CIRÚRGICA
}

Paloma Helena Sanches da Silva ${ }^{1}$; Amanda Oliveira Paraguassú ${ }^{2}$ Breno Curty Barbosa ${ }^{3}$; Nathália das Graças Dorneles Coelho ${ }^{3}$; Patrícia Maria Coletto Freitas ${ }^{4}$

1 Médica Veterinária autônoma com Residência em Clínica Cirúrgica em Animais de Companhia da Universidade Federal de Minas Gerais, Belo Horizonte - MG, Brasil (palomasanches.vet@gmail.com).

2 Graduanda do curso de Medicina Veterinária pela Universidade Federal de Minas Gerais.

3 Doutorando em Ciência Animal pela Universidade Federal de Minas Gerais.

3 Doutorando em Ciência Animal pela Universidade Federal de Minas Gerais.

4 Docente do Departamento de Clínica e Cirurgia Veterinárias da Universidade Federal de Minas Gerais.

\section{Recebido em: 06/04/2019 - Aprovado em: 10/06/2019 - Publicado em: 30/06/2019 DOI: 10.18677/EnciBio_2019A15}

\begin{abstract}
RESUMO
A criação seletiva artificial na obtenção de raças de cães e gatos, induzida pelo homem, trouxe grandes impactos principalmente no que se refere ao bem-estar desses animais. Embora tenha gerado uma grande variedade de raças, as modificações feitas em suas estruturas anatômicas, implicou inevitavelmente em mudanças fisiológicas e comportamentais em algumas raças caninas e felinas, trazendo grandes consequências aos mesmos. Ao interferir na conformação anatômica dos ossos do crânio de cães e gatos, modificando a forma e o tamanho da cabeça, há um estreitamento das vias aéreas, dificultando a passagem de ar. Consequentemente, desordens respiratórias acometeram os animais de focinho curto ocasionando a Síndrome Obstrutiva das Vias Aéreas do Braquicéfalo (Brachycephalic Obstructive Airway Syndrome - BOAS) ou Síndrome Braquicefálica. Essa síndrome é caracterizada principalmente por desordens das vias respiratórias, que levam a obstrução das mesmas de forma progressiva. As anormalidades que as compõe são compostas de alterações anatômicas classificadas como primárias e secundárias que podem ser tratadas se identificadas, embora nem todas sejam passíveis de correção cirúrgica. É uma doença progressiva, levando a alterações secundárias que podem ser irreversíveis ao animal. Tais alterações são consequências de um processo de obstrução crônica provocado pelas anormalidades primárias da síndrome. Em função da importância de seu conhecimento pelo médico veterinário, este trabalho objetivou realizar uma revisão de literatura direcionada principalmente para as principais anormalidades anatômicas das vias respiratórias que contribuem para a Síndrome Obstrutiva das vias Aéreas Braquicefálicas, e suas respectivas indicações cirúrgicas.
\end{abstract}

PALAVRAS-CHAVE: Braquicefálo; Cão; Obstrução. 


\title{
ANATOMICAL ABNORMALITIES OF THE BRACHYCEPHALIC RESPIRATORY WAYS AND ITS MAIN TECHNIQUES OF SURGICAL CORRECTION -
}

\begin{abstract}
Artificial selective breeding of man-induced breeds of dogs and cats has had major impacts mainly on the welfare of these animals. Although it spawned a wide variety of breeds, the modifications made in their anatomical structures inevitably entailed physiological and behavioral changes in some canine and feline breeds, bringing great consequences to them. By interfering in the anatomical conformation of the skull bones of dogs and cats by modifying the shape and size of the head, there is a narrowing of the airways, making it difficult to pass air. Consequently, respiratory disorders affected the animals with short snout causing the Brachycephalic Airway Obstruction Syndrome (Brachycephalic Obstructive Airway Syndrome - BOAS) or Brachycephalic Syndrome. This syndrome is characterized mainly by disorders of the airways, which lead to the obstruction of the same of progressive form. The abnormalities that compose them are composed of anatomical changes classified as primary and secondary that can be treated if identified, although not all of them can be corrected surgically. It is a progressive disease, leading to secondary changes that may be irreversible to the animal. Such changes are consequences of a process of chronic obstruction caused by the primary abnormalities of the syndrome. Due to the importance of its knowledge by the veterinarian, this work aimed to perform a literature review aimed mainly at the main anatomical abnormalities of the airways that contribute to the Brachycephalic Airway Obstructive Syndrome, and their respective surgical indications.
\end{abstract}

KEYWORDS: Brachycephalic; Dog; Obstruction.

\section{INTRODUÇÃO}

As raças que apresentam o comprimento do focinho reduzido, assim conhecido os animais braquicefálicos, tendem a ter um maior risco em desenvolver a Síndrome Obstrutiva das vias Aéreas Braquicefálicas ou Síndrome Braquicefálica (PACKER et al., 2015; LIU et al., 2017a). Essa particularidade dos braquicéfalos, na maioria das vezes, é desconhecida pelo público que só percebe ser um agravante quando esses animais começam a sinalizar os sintomas desencadeados pelas alterações anatômicas respiratórias presentes. Algumas das raças englobam o Bulldog inglês, Bulldog francês, Pug, Chihuahua, Cavalier King Charles Spaniel, Boston Terrier, Pequinês, Shih-tzu e Lhasa Apso (OECHTERING, 2010).

A síndrome pode ter componentes primários os quais envolvem principalmente narinas estenosadas, palato mole alongado e espessado, macroglossia, conchas nasais hipertrofiadas ou aberrantes e hipoplasia de traqueia (EMMERSON, 2014; OECHTERING, 2016; DUPRE; HEIDENREICH, 2016); e secundários como tonsilas palatinas evertidas, eversão de sáculos laríngeos e colapso de laringe avançado (EMMERSON, 2014; OECHTERING, 2016; DUPRE; HEIDENREICH, 2016).

Sinais clínicos envolvem as vias aéreas anteriores nas quais o fluxo aéreo fica comprometido, resultando em uma dispneia inspiratória obstrutiva, além de sintomas gastrointestinais poderem estar presente (DUPRE; HEIDENREICH, 2016). Fatores predisponentes como obesidade, clima do ambiente no qual o animal está inserido, e a atividade física ou exercício ao qual o animal é submetido podem contribuir para a ocorrência da síndrome (PACKER et al., 2015). 
Uma avaliação externa das narinas, do padrão respiratório apresentado pelo paciente, bem como a avaliação de todas as vias respiratórias com o auxílio dos exames de imagem são fundamentais para um diagnóstico conclusivo.

O tratamento consiste basicamente na correção cirúrgica das principais anormalidades anatômicas apresentadas passíveis de correção (EMMERSON, 2014). Algumas técnicas cirúrgicas compreendem a alaplastia (DUPRE; HEIDENREICH, 2016; MAZZARO; LEON-ROMAN, 2016), estafilectomia (HEFFERNAN et al., 2016; DUPRE; HEIDENREICH, 2016), tonsilectomia (COOK et al., 2015), saculectomia (HUGHES et al., 2018), ressecção da dobra ariepiglótica (FOWLER; BRIGHT, 2008), lateralização cricoaritenóide combinada a caudolateralização tireoaritenóide (WHITE, 2012) e traqueostomia permanente (GOBBETTI et al., 2018).

Uma combinação da terapia medicamentosa associada a diminuição dos fatores predisponentes também faz parte do tratamento (DUPRE; HEIDENREICH, 2016). Parece apresentar prognóstico favorável quando as anormalidades passíveis de correção cirúrgica são reconhecidas e tratadas precocemente (EMMERSON, 2014; LIU et al., 2017a).

\section{SÍNDROME OBSTRUTIVA DAS VIAS AÉREAS BRAQUICEFÁLICAS}

Consiste de uma ou mais combinação de anormalidades anatômicas e funcionais desencadeando predominantemente sinais de obstrução de vias aéreas devido a conformação do crânio desses animais (DUPRE; HEIDENREICH, 2016).

Como característica do crânio das raças braquiocefálicas, o crânio mostra-se mais comprido que a face (LIU et al., 2017b). Notavelmente, a região frontal é larga, bastante convexa e consequentemente, o desenvolvimento dos tecidos moles da região da cabeça e da face, bem como das estruturas da cavidade oral, não é proporcionalmente reduzido, não conseguindo assim, acompanhar o crânio encurtado dessas raças (PACKER et al., 2015; PICHETTO et al., 2015).

Os componentes da Síndrome podem ser divididos em primários ou secundários (OECHTERING et al., 2016). Os componentes classificados como sendo primários são aqueles responsáveis por provocar um aumento na resistência do fluxo aéreo no interior das vias respiratórias anteriores (FINDJI; DUPRE, 2013). Narinas estenosadas, palato mole alongado podendo também estar espessado, e hipoplasia de traqueia compõem as principais alterações primárias. Macroglossia e cornetos nasais hipertrofiantes ou aberrantes também tem sido reconhecidos como anormalidades primárias (OECHTERING et al., 2016; DUPRE; HEIDENREICH, 2016; FAWCETT et al., 2018).

Alterações adquiridas que resultam do crescente esforço inspiratório gerado pela obstrução crônica das vias aéreas, provocada pelas anormalidades primárias da Síndrome caracterizam as anormalidades secundárias (EMMERSON, 2014). Tais alterações envolvem principalmente tonsilas palatinas evertidas (COOK et al., 2015; LIU et al., 2017b; FAWCETT et al., 2018) e eversão de sáculos laríngeos e outros estágios do colapso de laringe (DUPRE; HEIDENREICH, 2016; FAWCETT et al., 2018.

\section{ANORMALIDADES PRIMÁRIAS}

A estenose das narinas é representada por uma malformação congênita das cartilagens nasais, as quais são caracterizadas pelo seu espessamento e por se colabarem medialmente dificultando a passagem do ar (DUPRE; HEIDENREICH, 2016). Devido ao estreitamento do orifício nasal que ocasiona uma diminuição da 
passagem do ar pelas vias respiratórias, tem-se também um comprometimento da função termorreguladora do órgão. Consequentemente, os animais portadores da síndrome braquicefálica apresentam dificuldade em regular sua temperatura corpórea em função da deficiência na liberação de calor, podendo vir a óbito, principalmente quando em condições ambientais de temperatura elevadas (OECHTERING et al., 2007; DAVIS et al., 2017). Além disso, o constante esforço inspiratório em detrimento ao acentuado estreitamento das narinas pode causar um aumento da pressão negativa na região de faringe e de traqueia, levando a uma inflamação das vias aéreas, podendo resultar em colapso das estruturas locais (DUPRE; HEIDENREICH, 2016).

O palato mole alongado é a deformidade mais comumente relatada em cães portadores da síndrome braquicefálica (BOFAN et al., 2015).

Normalmente, o palato mole é uma continuidade do palato duro e que se estende até a extremidade da epiglote, a qual está localizada na entrada da laringe.

Nos braquicefálicos, quando o prolongamento desta estrutura está presente, ocorre um deslocamento do mesmo sobre a rima da glote durante a inspiração, interferindo negativamente na respiração (MATHEWS, 2012). Para visualizar tal anormalidade, é necessária anestesia geral do paciente e uso de um laringoscópio. Uma imagem radiográfica da região cervical também pode evidenciar o palato mole excessivamente longo e até mesmo espessado (CANOLA et al., 2018). Considerase tal estrutura alongada, caso a mesma estiver ultrapassando a borda da epiglote, uma vez que normalmente, ele não causa obstrução do fluxo de ar na região (MATHEWS, 2012).

Já se sabe que não somente o fato de apresentar um prolongamento, mas também a espessura encontrada no palato mole desses animais pode contribuir para obstrução do fluxo aéreo e consequentemente, interferir de forma negativa na respiração dos animais (PICHETTO et al., 2015). Essas características do palato mole induzem a uma inflamação da faringe, tonsilas e até mesmo da laringe, onde seu prolongamento excessivo sobrepõe-se a laringe causando tumefação da mesma e com isso, agravando ainda mais a passagem de ar (PICHETTO et al., 2015; BOFAN et al., 2015).

A hipoplasia de traqueia é considerada um componente primário da Síndrome e quando presente pode ocorrer concomitantemente às outras anormalidades como estenose de narinas e ao palato mole prolongado (OECHTERING, 2010). A traqueia hipoplásica é caracterizada pelo seu diâmetro reduzido em toda sua extensão (MILLER; GANNON, 2015). Embora o diâmetro reduzido ao longo do seu lúmen resulte numa resistência à passagem de ar, alguns autores alegam que tal alteração exerce influência mínima sobre a Síndrome Braquicefálica, como no relato descrito por Allemand et al. (2013), no qual foi possível obter resultado clínico satisfatório após o tratamento cirúrgico de anormalidades passíveis de correção em um cão, no caso, com palato mole alongado e sáculos laríngeos evertidos, mesmo com a presença da traqueia hipoplásica.

A hipertrofia das conchas ou cornetos nasais tem sido relatada em raças braquicefálicas como sendo uma alteração anatômica dos cornetos nasais que pode contribuir para a ocorrência da síndrome em questão (OECHTERING et al., 2016). Essa condição pode ser diagnosticada com a tomografia computadorizada ou com a rinoscopia (OECHTERING et al., 2016).

Anatomicamente, os cornetos são estruturas intranasais que se projetam da parede lateral da cavidade nasal em direção ao septo, permitindo a passagem do ar nos meatos (OECHTERING et al., 2016). Quando proeminentes, isto é, 
apresentando hipertrofia, podem contribuir para obstrução do fluxo de ar ao provocar pontos de contato entre as mucosas da região, aumentando sua resistência na via aérea (SCHUENEMANN; OECHTERING, 2014; HOSTNIK et al., 2017). A presença desses cornetos que crescem com uma configuração aberrante e malformada está relacionada ao desenvolvimento dos tecidos moles de uma cavidade nasal reduzida seletivamente em decorrência do encurtamento craniano encontrado nas raças braquicefálicas. Esses cornetos aberrantes podem ocupar o meato da nasofaringe limitando ainda mais a passagem de ar na região (SCHUENEMANN et al., 2016).

Macroglossia é um termo utilizado na medicina humana que descreve uma língua comprida ao projetar-se além dos dentes durante o momento de repouso da cavidade oral (BALAJI, 2013). Entretanto, apesar desta definição ser mais exata ao referido termo, a macroglossia também tem sido citada recentemente na literatura veterinária, como sendo um dos componentes importantes na Síndrome Braquicefálica (DUPRE; HEIDENREICH, 2016; HOSTNIK et al., 2017; LIU et al., 2017b; FAWCETT et al., 2018), por ser comumente observada nos animais de focinho curto os quais apresentam uma língua aparentemente espessa e comprida. O que acontece nos braquicefálicos, é que a língua é desproporcional ao espaço reduzido que ocupa na cavidade oral, dando a impressão de ser uma estrutura grande (PACKER et al., 2015). Com isso, contribui para um deslocamento dorsal do palato mole, prejudicando a passagem de ar na via respiratória (FOX, 1963).
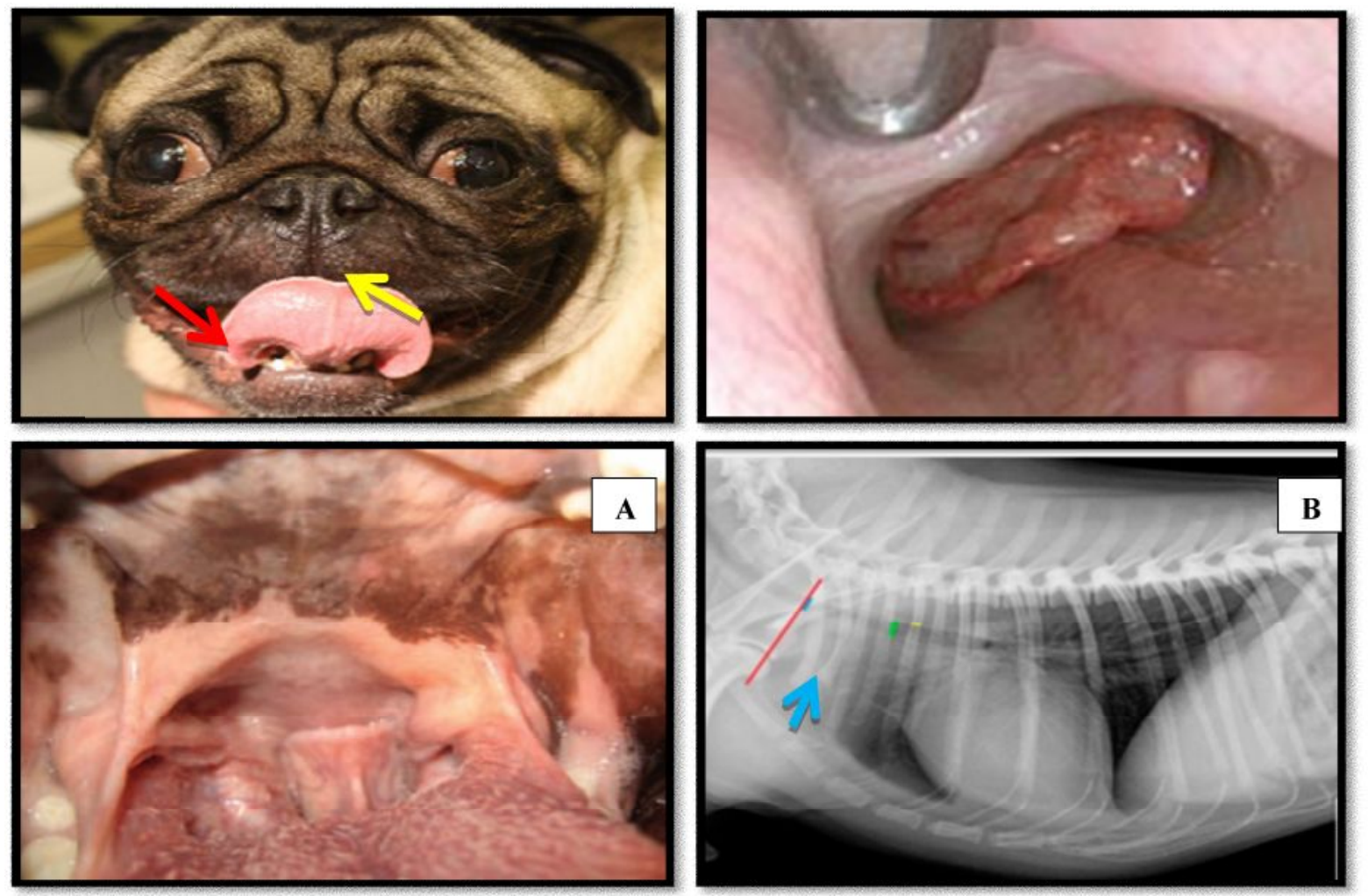

Figura 1: Anormalidades primárias dn Síndrome Braquicefálica. A: Estenose do Narinas (seta amarela) e Macroglo C (seta vermelha) (Fonte: Packer; Tiv D 2015). B: Presença de corneto nasal caudal aberrante (CAT - Caudal Aberrants Turbinates) em região de meato nasal (Fonte: Oechtetering, 2010). C: Palato mole alongado ocluindo entrada da glote (Fonte: Trappler; Moore, 2011). D: Imagem radiográfica na posição latero-lateral evidenciando o colapso de traqueia (seta azul) (Fonte: Souto et al., 2015). 


\section{ANORMALIDADES SECUNDÁRIAS}

Tonsilas palatinas estão localizadas no interior das criptas tonsilares, imediatamente caudais às bordas caudolaterais do palato mole (MATTEWS, 2012; LODATO; MAUTERER, 2014). Considerada anormalidade secundária da Síndrome gerada como consequência do constante esforço inspiratório de estenoses mais rostrais (LODATO; MAUTERER, 2014) e caracterizadas por tornarem-se edemaciadas e protuídas para dentro da cavidade oral, podendo contribuir para o aumento da resistência do ar na região da faringe (BOFAN et al., 2015).

Normalmente os sáculos laríngeos são estruturas anatômicas presentes na entrada da laringe, localizadas entre as cordas vocais e vestibulares $(\mathrm{KOCH}$ et al., 2003). Uma vez que a mucosa dos sáculos everte, é possível visualizá-la com exame de laringoscopia (CANTATORE et al., 2012). Os sáculos laríngeos evertidos são considerados o primeiro estágio do colapso de laringe ou estágio I (CACCAMO et al., 2014).

Quando presentes estão associados a um histórico de obstrução crônica que decorre de estenoses mais rostrais, levando a um aumento de pressão negativa anormal na região da faringe. Por isso, quando presentes são considerados anormalidades secundárias da síndrome braquicefálica (FAWCETT et al., 2018).

Para ocorrer o colapso da laringe no cão, três estágios precisam ser alcançados, segundo a descrição de Leonard (1960) seguida até os dias atuais. Após a eversão dos sáculos laríngeos o qual configura o estágio I do colapso, havendo progressão da dispneia inspiratória que torna a pressão intraglótica ainda mais negativa, os processos das cartilagens aritenóides perdem a rigidez e são tracionados medialmente resultando em deformidade permanente da cartilagem. Com isso, chega-se aos estágios mais avançados do colapso (FAWCETT et al., 2018).

Tais estágios, II e III envolvem os processos cuneiforme e corniculado, respectivamente. Os processos cuneiformes que antes, anatomicamente, estendiam-se à região caudolateral da faringe na inspiração, agora sofrem um deslocamento medial caracterizando o segundo estágio do colapso. Quando há desvio medial dos processos corniculados, ocorre uma perda do arco dorsal da rima glótica, caracterizando o estágio mais avançado do colapso, o grau III (LEONARD, 1960). Uma vez que esses processos são desviados, podem até se sobreporem e um colapso de laringe então, é gerado, podendo ser fatal (LEONARD, 1960; CANTATORE et al., 2012). Cães da raça Pug são frequentemente mais propensos a sofrerem colapso de laringe que Buldogues (HAIMEL; DUPRE, 2015). 

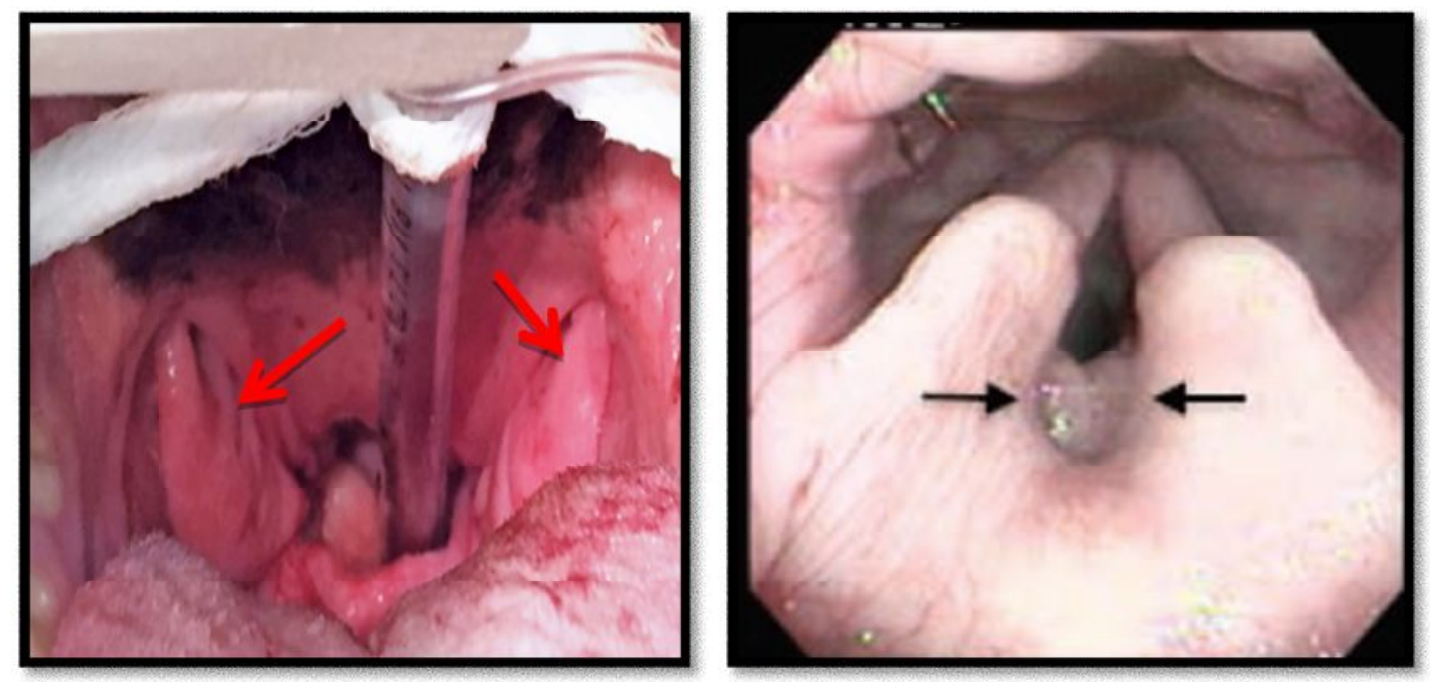

Figura 2: $\dot{A}$ esquerda, mucosa das tonsilas palatinas edemaciadas e evertidas (setas vermelhas) (Fonte Phillips; Jaeger, 2016:). À direita, sáculos laríngeos evertidos ou estágio I do colapso de laringe (setas pretas). (Fonte: Trappler; Moore, 2011).

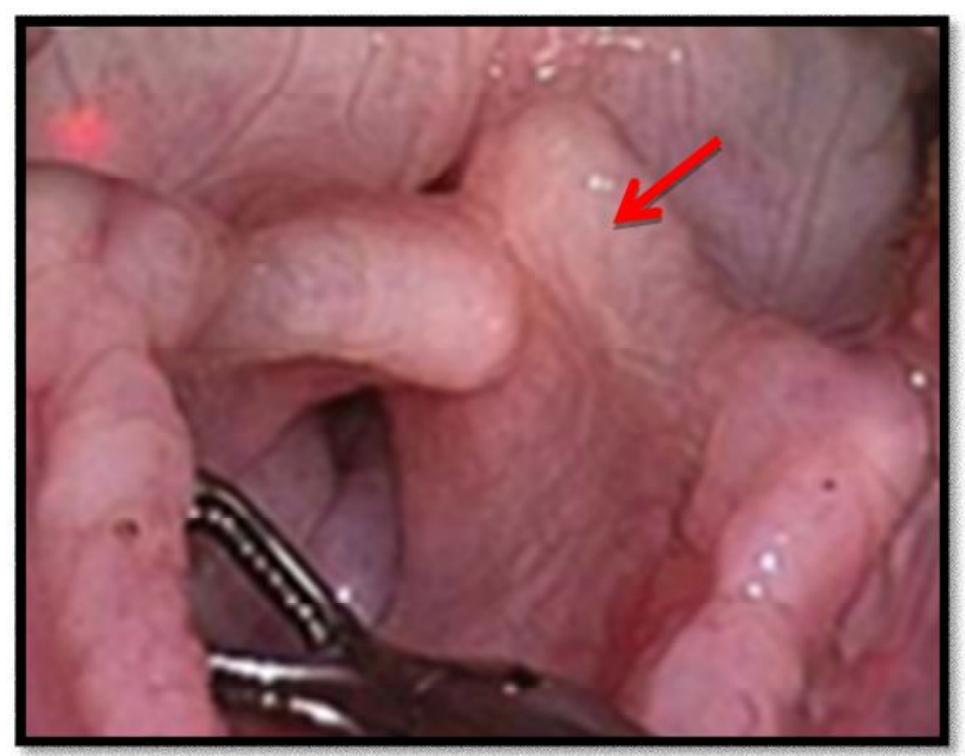

Figura 3: Estágio II do colapso de laringe. Note o desvio medial dos processos cuneiformes (seta vermelha) e os sáculos laríngeos evertidos. (Fonte: Packer; Tivers, 2015). 


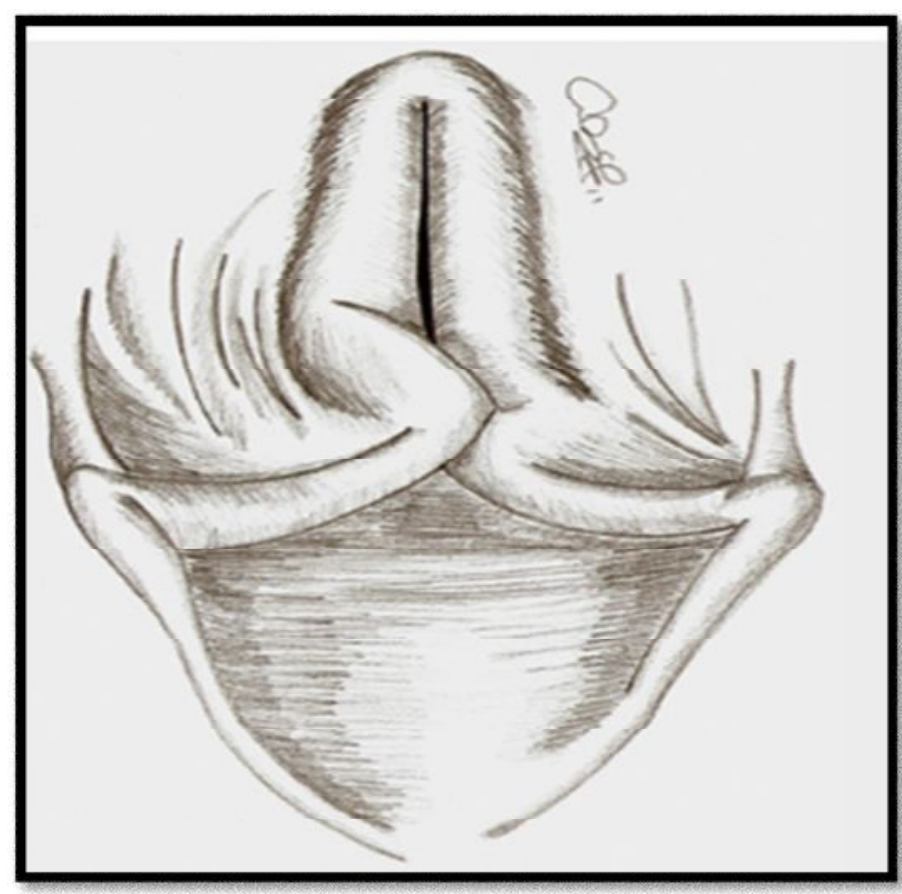

Figura 4: À direita, estágio III do colapso de laringe. Notar o desvio medial dos processos corniculados das cartilagens aritenóides. (Fonte: Miller; Gannon; 2015).

\section{TÉCNICAS CIRURGICAS DESCRITAS PARA TRATAMENTO DA SÍNDROME BRAQUICEFÁLICA}

O tratamento da Síndrome consiste de uma associação entre o uso de terapia medicamentosa, diminuição dos fatores de risco e o uso de técnicas cirúrgicas às anormalidades anatômicas presentes que são passíveis de correção (EMMERSON, 2014; DUPRE; HEIDENREICH, 2016).

Vale ressaltar que as anormalidades anatômicas das vias respiratórias devem ser identificadas e corrigidas precocemente para obtenção de melhores resultados (EMMERSON, 2014), uma vez que já tem sido demonstrado que o comprometimento respiratório ainda pode estar presente nos casos em que há anormalidades, que não foram devidamente identificadas e tratadas como a presença das conchas nasais aberrantes ou a presença de alterações que não são passíveis de correção cirúrgica, como a macroglossia e a hipoplasia de traqueia (LIU et al., 2017b).

As principais técnicas cirúrgicas visam corrigir algumas das anormalidades conhecidas como narinas estenosadas, palato mole alongado e espessado, cornetos nasais aberrantes, tonsilas palatinas evertidas, sáculos laríngeos evertidos e outros estágios avançados do colapso de laringe, todos com o intuito de diminuir a resistência da passagem do ar nas vias aéreas (DUPRE; HEIDENREICH, 2016).

Nos casos de estenose de narinas, as técnicas cirúrgicas englobam principalmente a alaplastia cujo intuito é aumentar o tamanho das narinas estenosadas, contribuindo para evitar o desenvolvimento de alterações secundárias da Síndrome (MAZZARO; LEON-ROMAN, 2016). Esta técnica pode ser executada pelas seguintes abordagens: alaplastia por ressecção em cunha da asa da narina ou alaplastia por ressecção com punch (DUPRE; HEIDENREICH, 2016). 
A ressecção em cunha é a técnica mais abordada na literatura veterinária (BOFAN et al., 2015). Cria-se uma incisão com a lâmina de bisturi sobre a asa da narina, a qual deve ser em forma de cunha, podendo ser vertical, horizontal ou lateral (DUPRE; HEIDENREICH, 2016), permitindo assim, a remoção de um segmento da asa, seguida do fechamento primário do defeito com fio monofilamentar. O mesmo procedimento é executado na narina contralateral, respeitando a simetria das narinas. A incisão deve ser profunda a ponto de atingir a prega alar para que assim ocorra uma abertura funcional das narinas (BOFAN et al., 2015). Essa técnica pode ser realizada também com o uso de laser $\mathrm{CO}_{2}$ apresentando a vantagem de causar hemorragia mínima, otimização do tempo de cirurgia e anestesia quando comparada ao método convencional, além de ser mais confortável ao paciente no pós-operatório, uma vez que não se utiliza fios cirúrgicos e diminui o tempo de recuperação (LODATO; MAUTERER, 2014).

A ressecção das narinas com o uso de um punch também pode ser realizada. Esta técnica permite a remoção de mesmo tamanho dos fragmentos em ambas narinas a partir de uma ferramenta dermatológica, com a vantagem de garantir um bom resultado simétrico e cosmético (TROSTEL; FRANKEL, 2010).

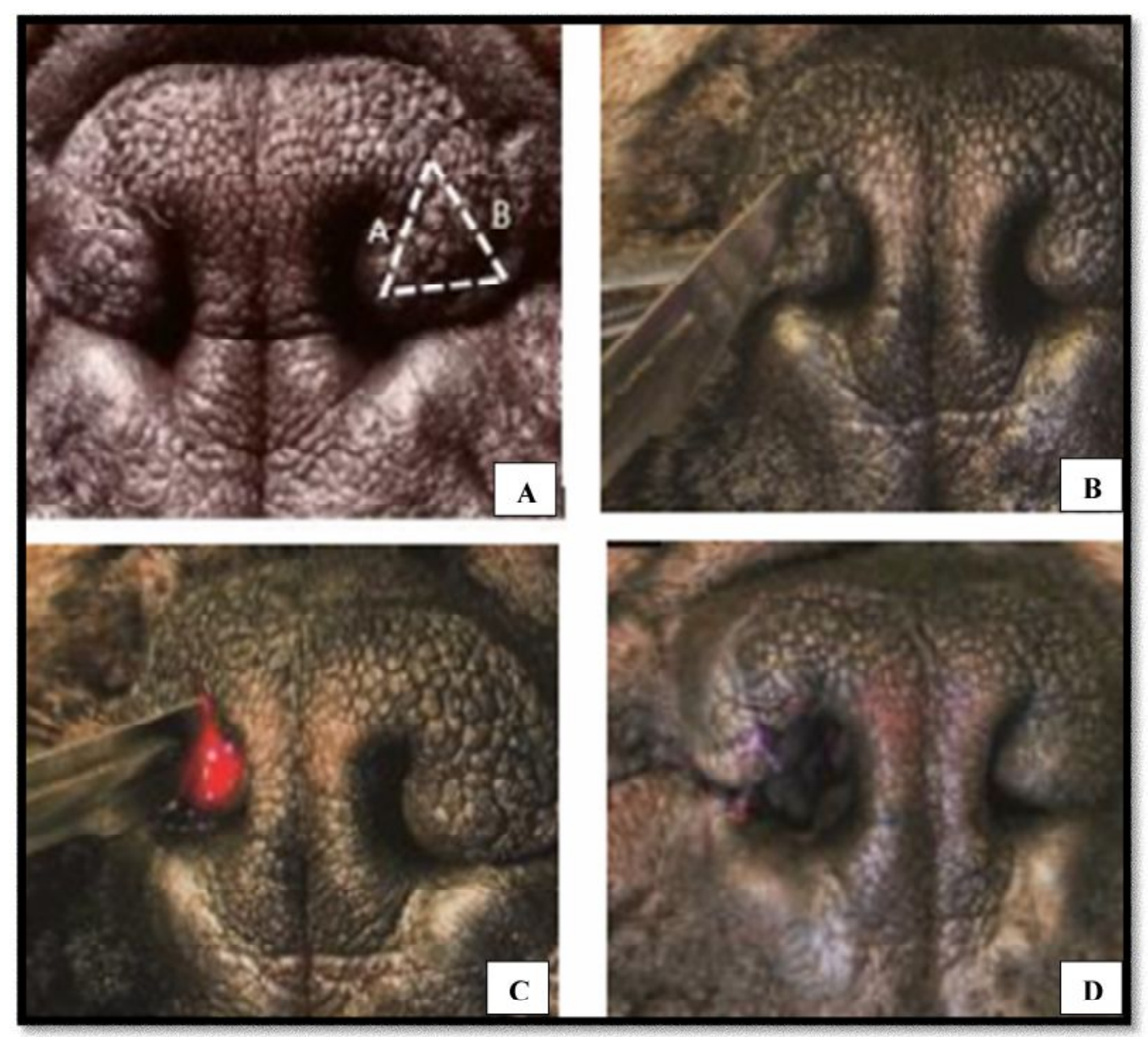

Figura 5: Demonstração de rinoplastia ou alaplastia. A: marcação da incisão em triangular ou em cunha demonstrada na asa da narina; $\mathbf{B}$ : incisão realizada com lâmina de bisturi № 11; C: incisão aprofundada para alcançar prega alar; D: sutura das bordas com fio absorvível e abertura das narinas. Fonte: (Finji; Dupre, 2013). 
Quando o palato mole alongado está presente, a estafilectomia ou palatoplastia é indicada (FAWCETT et al., 2018). O procedimento consiste na ressecção da porção alongada do palato mole com a finalidade de encurtá-lo ao nível da região caudal das tonsilas palatinas, de forma que sua borda caudal livre fique apoiada sobra à extremidade epiglótica minimizando a possibilidade de edema, e logo, facilitando a passagem de ar sem obstrução da rima da glote na inspiração (CANTATORE et al., 2012).

A estafilectomia pode ser executada utilizando-se bisturi ou tesoura, além de pinças hemostáticas e aposição das mucosas da orofaringe e nasofaringe, em padrão simples contínuo com fio absorvível após a ressecção do tecido em excesso (LODATO; MAUTERER, 2014). A palatoplastia também pode ser realizada utilizando-se eletrocautério monopolar, além de laser como, por exemplo, de $\mathrm{CO}_{2}$ (KIRSCH et al., 2019) o qual pode apresentar algumas vantagens em relação à técnica convencional como a redução no tempo de duração da cirurgia, tempo de recuperação mais rápido e menor formação de edema local devido a pouca manipulação tecidual, diminuindo assim, as chances de complicações no pósoperatório imediato.

Outra técnica para correção de palato mole alongado é a palatoplastia de flap ou aba dobrada, sendo indicada principalmente quando o palato também é espessado (FINDJI; DUPRE, 2013). Este procedimento parece apresentar vantagem adicional à técnica convencional, uma vez que o objetivo envolve não somente 0 encurtamento do palato, mas também de torná-lo mais fino, sendo possível assim promover uma melhor desobstrução da faringe e então, garantir uma passagem de ar normal nas regiões da nasofaringe e da orofaringe. Para isso, é criada uma incisão trapezoidal sobre uma porção da mucosa do palato mole, seguida da dobra do palato em si (FINDJI; DUPRE, 2013). Vale ressaltar, que é uma técnica mais complexa e que demanda mais tempo de cirurgia quando comparada à técnica de ressecção convencional (FINDJI; DUPRE, 2013).

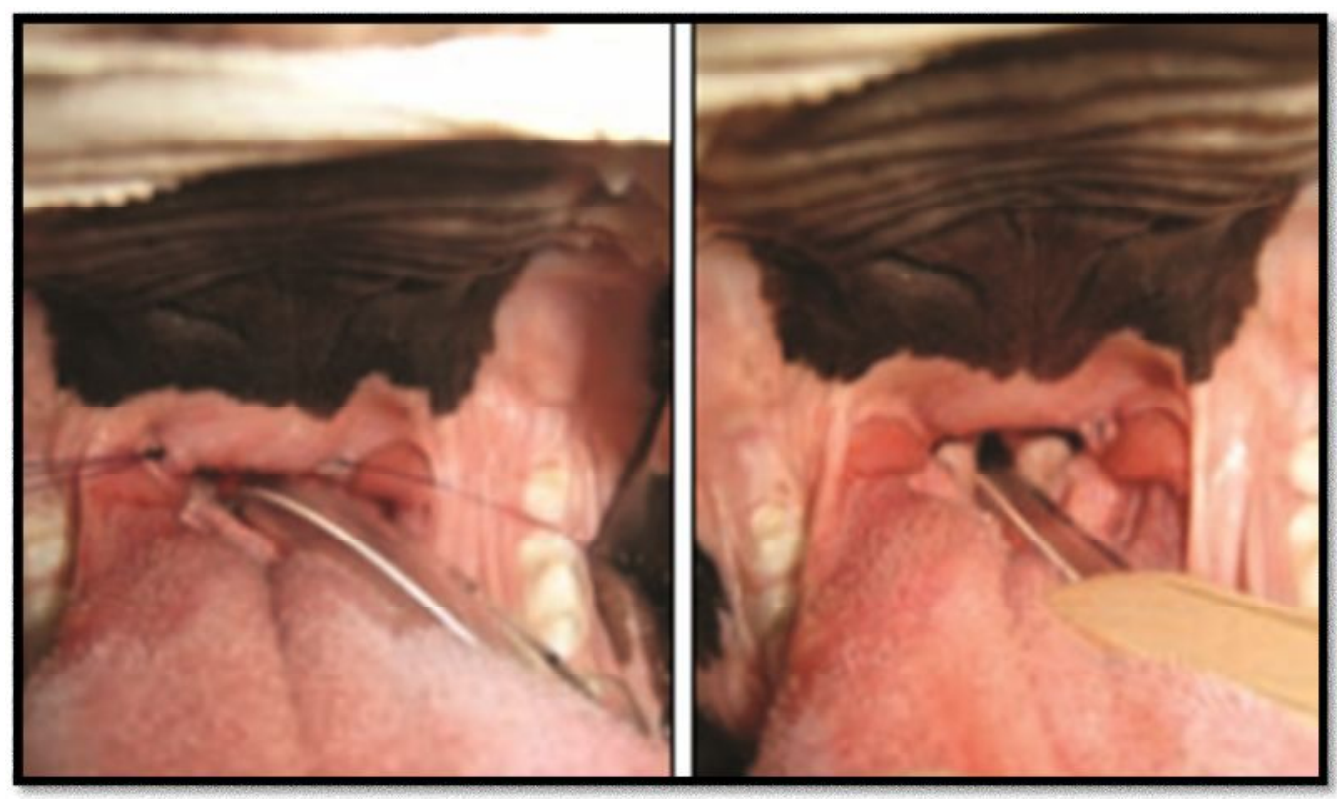

Figura 6: Demonstração da técnica de estafilectomia tradicional. Imagem à esquerda com uso de fios cirúrgicos para reparo e tesoura para ressecção do tecido em excesso. À direita, sutura do palato mole alongado finalizada e visualização da entrada da glote. Fonte: (Fonte: Trappler; Moore, 2011) 


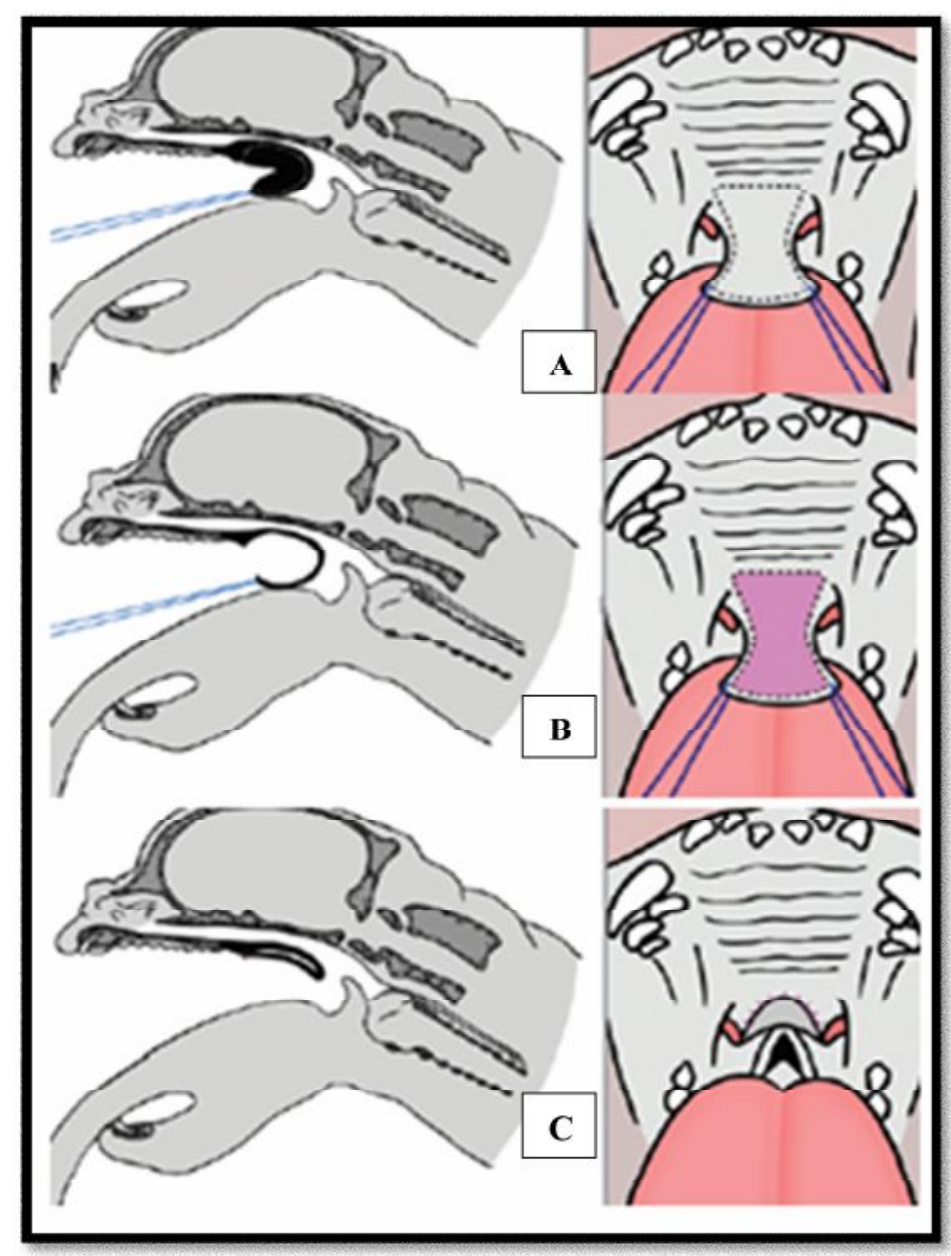

Figura 7: Demonstração da técnica de estafilectomia em flap dobrado. A: palato mole alongado e espessado com fios de reparo, à esquerda. B: criação e remoção do flap sobre palato mole espessado e alongado. C: flap de mucosa dobrado e suturado. (Fonte: Findji; Dupre, 2013).

Para correção das conchas nasais malformadas, pode ser empregada a técnica de turbinectomia assistida a laser diodo guiada por um rinoscópio (SCHUENEMANN; OECHTERING, 2016). Apresenta vantagem de ser uma técnica minimamente invasiva e permite a ressecção dos cornetos nasais aberrantes rostral e caudal (OECHTERING el al., 2016), demonstrado bons resultados ao melhorar o fluxo de ar intranasal (SCHUENEMANN et al., 2016; LIU et al., 2018). A cirurgia só é indicada se os cornetos hipertrofiados estiverem causando pontos de contato entre as mucosas e obstrução da via aérea (SCHUENEMANN et al., 2016). 


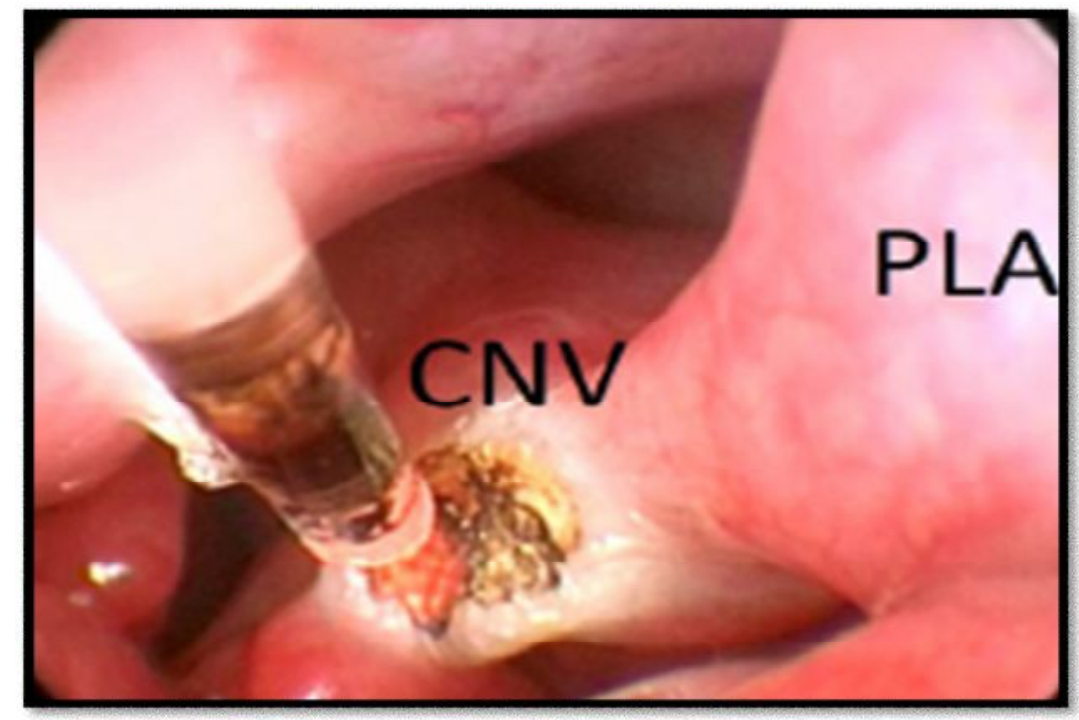

Figura 8: Imagem endoscópica demonstrando Turbinectomia a laser guiado por rinoscópio para ressecção de concha nasal ventral aberrante (CNV). PLA: Prega alar. (Fonte: OECHTERING et al., 2014).

Tonsilectomia é o nome dado à técnica cirúrgica para remoção das tonsilas palatinas alteradas (LODATO; MAUTERER, 2014). Entre outras indicações, pode ser aplicada no tratamento da Síndrome Braquicefálica, quando a eversão tonsilar em decorrência do edema formado na mesma, contribui para aumentar a resistência do fluxo de ar resultando em algum grau de obstrução na região da faringe (BOFAN et al., 2015; COOK et al., 2015). Entretanto, alguns autores contraindicam sua execução alegando ser desnecessária nestes casos, ao predispor a uma inflamação na região durante o pós-operatório, podendo resultar em complicações ao animal; ainda afirmam, que a correção das anormalidades primárias concomitantes é suficiente, pois o edema nas tonsilas tende a ser reduzido em seguida, retornando espontaneamente a mucosa para o interior da fossa tonsilar, desprezando a necessidade de intervenção cirúrgica (KOCH et al., 2003; MATHEWS, 2012).

Para a remoção das tonsilas palatinas evertidas, pode-se empregar o uso do bisturi, tesoura, eletrocautério monopolar, laser de $\mathrm{CO}_{2}$ e até mesmo um dispositivo selador de vasos bipolares (COOK et al., 2015). Pode ou não necessitar de suturas com fio absorvível após a ressecção (LODATO; MAUTERER, 2014; BELCH et al., 2016). 


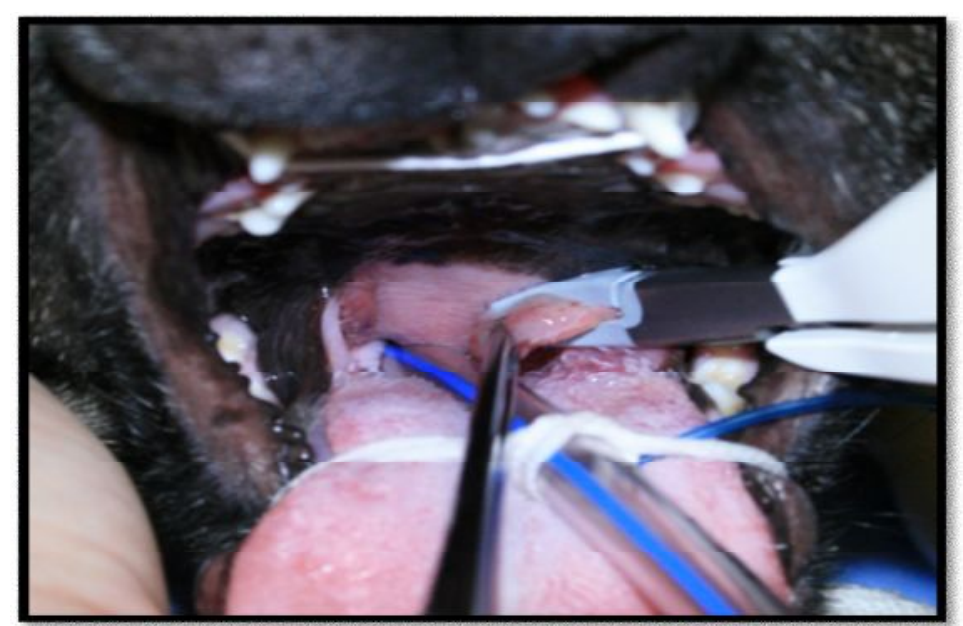

Figura 9: Demonstração de tonsilectomia após eversão de tonsilas palatinas com auxílio de um dispositivo selador bipolar. (Fonte: COOK et al., 2015).

A técnica para excisão dos sáculos ou saculectomia consiste na amputação dos mesmos (PACKER; TIVERS, 2015; HUGHES, et al., 2018) e pode ser feita utilizando-se tesouras Metzenbaum devido a localização anatômica dos sáculos (ALLEMAND et al., 2013). Uma vez corrigidos, permitem uma maior abertura da rima da glote permitindo melhor a passagem do ar (TORREZ; HUNT, 2006; RIECKS et al., 2007; TROSTEL; FRANKEL, 2010). Entretanto, recentemente, um estudo realizado por Hughes et al. (2018), concluiu que a saculectomia apesar de trazer resultados favoráveis pode também, resultar em complicações de leves a graves no pós-operatório imediato, o que sugere que a indicação de executar a técnica deve ser antes ponderadamente analisada.

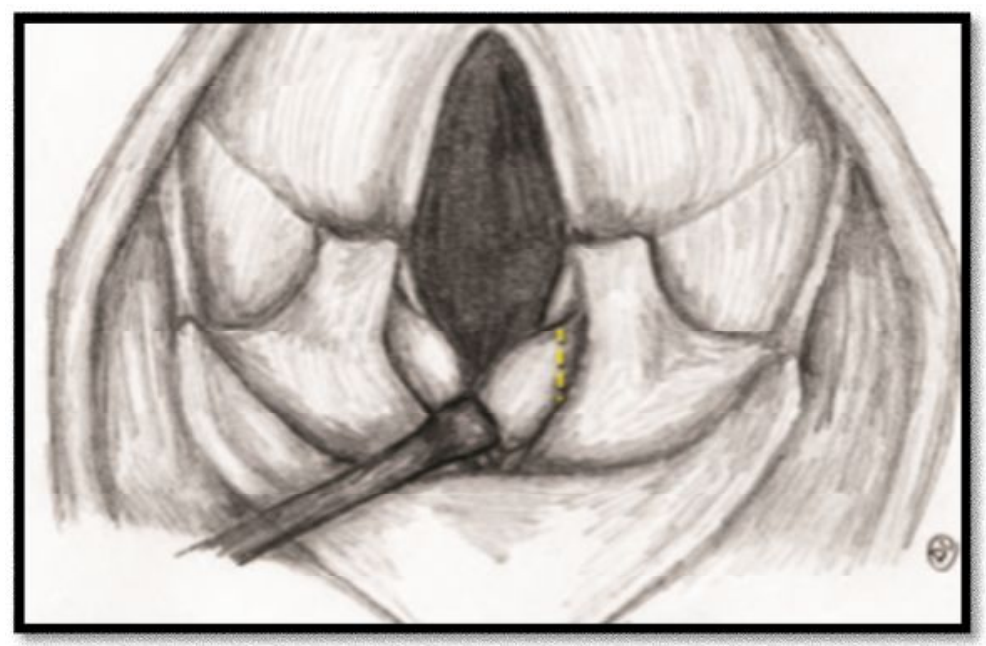

Figura 10: Saculectomia com auxílio de pinça allis para tração e posterior ressecção com tesoura. Fonte: (LODATO; HEDLUND, 2014).

Das anormalidades anatômicas presentes, o colapso de laringe quando em estágios avançados pode ser uma condição desafiadora para tratamento (PACKER; TIVERS, 2015). Alguns casos relatados demonstram controvérsia quanto aos resultados após o emprego de técnicas corretivas para este tipo de anormalidade na Síndrome (WHITE, 2012). Há relatos em que a correção cirúrgica para o colapso 
laríngeo avançado tem apresentado um alto índice de complicações, além de ter trazido poucos benefícios no pós-operatório (PINK et al., 2006; GOBBETTI et al., 2018). Outros relatos demonstraram bons resultados em um período de cinco anos após a cirurgia (WHITE, 2012).

Algumas técnicas cirúrgicas são descritas na literatura, porém consideradas limitadas (PACKER; TIVERS, 2015). Tais técnicas, envolvem a ressecção da dobra ariepiglótica, a qual consiste na secção da dobra com tesoura, permitindo sua cicatrização por segunda intenção e logo, uma melhor abertura da laringe (FOWLER; BRIGHT 2008); lateralização cricoaritenóide combinada a caudolateralização tireoaritenóide que consiste em reposicionar a cartilagem aritenóide deformada para um melhor fluxo de ar (WHITE, 2012) e finalmente a traqueostomia permanente, técnica que permite a criação de um stoma permanente na traqueia facilitando assim, a passagem do ar (GOBBETTI et al., 2018).

Segundo Gobbetti et al. (2018), a traqueostomia permanente é uma opção de resgate, sendo o único recurso atual para tentar tratar animais com colapso de grau avançado, embora complicações como obstrução por muco, deiscência ou estenose do stoma também sejam esperadas nos braquicefálicos devido a seu pescoço curto e largo.

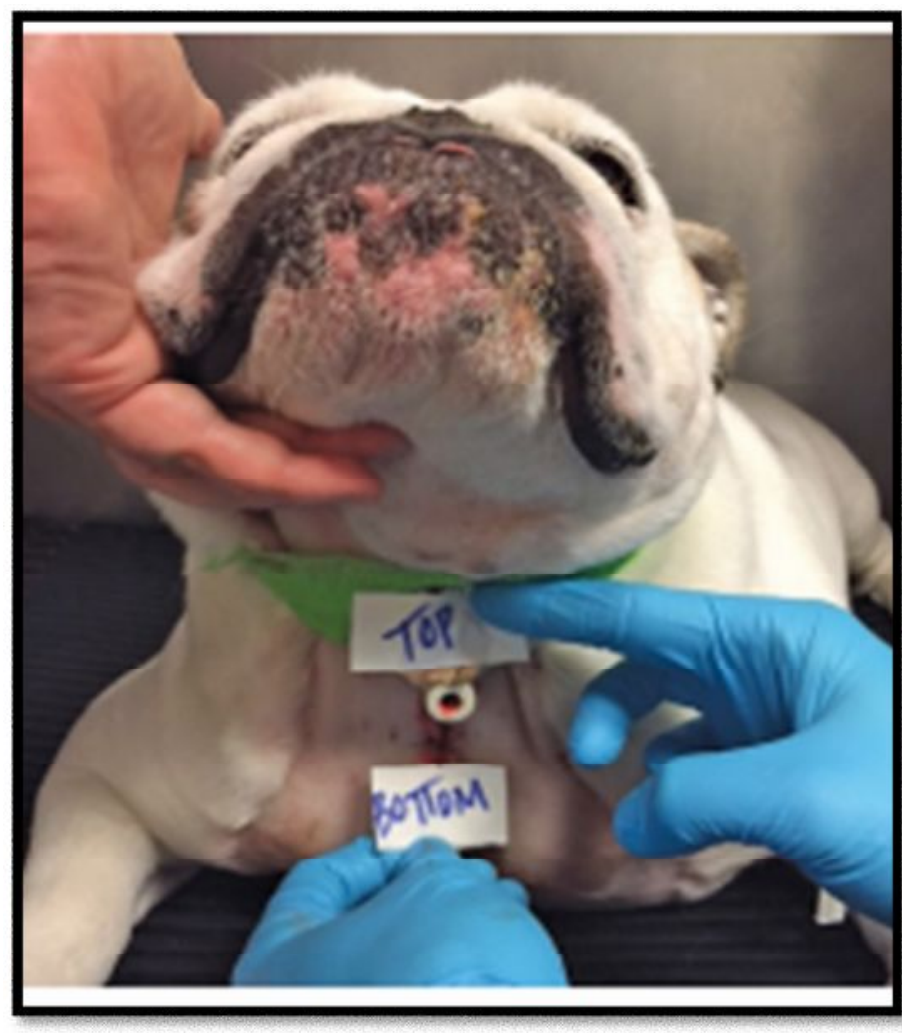

Figura 11: Traqueostomia em tubo demonstrada em cão da raça Buldogue com colapso de laringe avançado. (Fonte: PHILLIPS; JAEGER, 2016).

A eutanásia em alguns trabalhos também tem sido citada nos casos mais agravantes do colapso em que não se obtém sucesso com as técnicas descritas acima (PINK et al., 2006). 


\section{CONSIDERAÇÕES FINAIS}

A Síndrome Braquicefálica é uma condição progressiva que pode afetar gravemente a respiração do braquicefálico. Desta forma, cabe ao médico veterinário ter conhecimento das anormalidades anatômicas respiratórias que podem estar presentes, bem como identificá-las e indicar tratamento apropriado, principalmente àquelas passíveis de correção cirúrgica

\section{REFERÊNCIAS}

ALLEMAND, V.C.D.; QUIZANI, M.; BERL, C.A. Síndrome respiratória dos cães braquicefálicos: Relato de caso. Revista de educação continuada em Medicina Veterinária e Zootecnia do Conselhor Regional de Medicina Veterinária, v.11, n.2, p.42-47, 2013. Disponível em: <https://www.revistamvezcrmvsp.com.br/index.php/recmvz/article/view/16222/17088>

BALAJI, S.M. Glossectomia de redução para grandes línguas. Annals of Maxillofacial Surgery, v.3, n.2, p.167-172, 2013. Disponível em: $<$ http://www.amsjournal.com/article. $a s p ?$ issn=2231746; year $=2013$; volume $=3$; issue $=2$ ;spage $=167$; epage $=172$; aulast=Balaji $>$ doi: $10.4103 / 2231-0746.119230$

BELCH, A.; MATIASOVIC, M.; RASOTTO, R.; DEMETRIOU, J. Comparison of the use of LigaSure versus a standard technique for tonsillectomy in dogs. Veterinary Record, v.180, $\quad$ n.8, $2016 . \quad$ Disponível em:< https://veterinaryrecord.bmj.com/content/180/8/196> doi10.1136/vr.103873.

BOFAN, B.A.; IONASCU, I.; SONEA, A. Brachycephalic airway syndrome in dogs. Scientific Works. Series C. Veterinary Medicine, v. 61, n.1, p.103-112, 2015. Disponível em:

http://veterinarymedicinejournal.usamv.ro/pdf/2015/issue 1/Art18.pdf> doi: 10.1111/vsu.12608.

CACCAMO, R.; BURACCO, P.; LA ROSA, G.; CANTATORE, M.; ROMUSSI, S. Glottic and skull indices in canine brachycephalic airway obstructive syndrome. BMC Veterinary Research, v.10, n.12, p.1-12, 2014. Disponível em: $<$ http://www.biomedsearch.com/nih/Glottic-skull-indices-in-canine/24410902.html> doi:10.1186/1746-6148-10-12.

CANOLA, R.A.M.; SOUZA, M.G.; BRAZ, J.B.; RESTAN, W.A.Z.; YAMADA, D.I et al. Cardiorespiratory evaluation of brachycephalic syndrome in dogs. Pesquisa Veterinária Brasileira, v. 38, n. 6, p.1130-1136, 2018. Disponível em: <. http://dx.doi.org/10.1590/1678-5150-pvb-5376 doi:10.1590/1678-5150-pvb-5376

CANTATORE, M.; GOBBETTI, H.; ROMUSSI, S.; BRAMBILLA, L.; GIUDICE, C. et al. Medium term endoscopic assessment of the surgical outcome following laryngeal saccule resection in brachycephalic dogs. Veterinary Record, v.170, n.20, p.1-7, 2012. Disponível em: < https://www.ncbi.nlm.nih.gov/pubmed/22472536> doi: 10.1136/vr.100289 
COOK, D.A.; MOSES, P.A.; MACHIE, J.T. Clinical effects of the use of a bipolar vessel sealing device for soft palate resection and tonsillectomy in dogs, with histological assessment of resected tonsillar tissue, Australian Veterinary Journal, n.12, p.445-451, 2015. Disponível em: < https://doi.org/10.1111/avj.12384> doi: $\underline{10.1111 / \text { avj. } 12384}$

DAVIS, M.S.; CUMMINGS, S.L.; PAYTON, M.E. Effect of brachycephaly and body condition score on respiratory thermoregulation of healthy dogs. Journal of the American Veterinary Medical Association, v.251, n.10, p.1160- 1165, 2017. Disponível em: <https://www.ncbi.nlm.nih.gov/pubmed/26790550> doi:10.2460/javma.251.10.1160

DUPRÉ, G.; HEIDENREICH, D. Brachycephalic syndrome. Veterinary Clinics of North America: Small Animal Pratice, v.46, n.1, p.691-707, 2016. <Disponível em: <https://doi.org/10.1016/i.cvsm.2016.02.002> doi: 10.1016/j.cvsm.2016.02.002

EMMERSON, T. Brachycephalic obstructive airway syndrome: A growing problem. Journal of Small Animal Practice, v.55, n.11, p.543-544, 2014. Disponível em: < http://dx.doi.org/10.1111/jsap.12286> doi:10.1111/jsap.12286

FAWCETT, A.; BARRS, V.R.; AWAD, M.; CHILD, G.; BRUNEL, L. et al. Consequences and Management of Canine Brachycephaly in Veterinary Practice: Perspectives from Australian Veterinarians and Veterinary Specialists. Animals: an open access journal from MDPI, v.9, n.1, p.1-25, 2018. Disponível em: $<$ https://www.ncbi.nlm.nih.gov/pmc/articles/PMC6356869/pdf/animals-09-00003.pdf > doi: $\underline{10.3390 / a n i 9010003}$

FINDJI, L. DUPRE, G. Brachycephalic Syndrome: Innovative Surgical Techniques. Clinician's brief, v.11, n.6, p.79-85, 2013. Disponível em: < https://www.cliniciansbrief.com/article/brachycephalic-syndrome-innovative-surgicaltechniques>

FOWLER, J.D.; BRIGHT, R.M. Developmental Disorders - Brachycephalic Syndrome. In: MORGAN, R.V. Handbook of Small Animal Practice: Diseases of the Larynx and Pharynx, 5 ed. Tenneesse: Saunders, 2008, Cap 15 p.146-154. Disponível em: <https://doi.org/10.1016/B978-1-4160-3949-5.50019-4>

FOX, M.W. Developmental abnormalities of the canine skull. Canadian journal of comparative medicine and veterinary science, v.27, n.9, p. 219-222, 1963. Disponível em: https://www.ncbi.nlm.nih.gov/pmc/articles/PMC1583693/>

GOBBETTI, M.; ROMUSSI, S.; BURACCO, P.; BRONZO, V.; GATTI, S.; et al. Longterm outcome of permanent tracheostomy in 15 dogs with severe laryngeal collapsesecondary to brachycephalic airway obstructive syndrome. Veterinary Surgery, v.47, n.5, p.648-653, 2018. Disponível em: < https://www.ncbi.nlm.nih.gov/pubmed/29893060>doi: 10.1111/vsu.12903.

HAIMEL, G.; DUPRE, G. Brachycephalic airway syndrome: A comparative study between Pugs and French Bulldogs. Journal of Small Animal Practice, v.56, n.12, 
p. 714-719, 2015. Disponível em: https://www.ncbi.nlm.nih.gov/pubmed/26563910 <doi: 10.1111/jsap.12408.

HEFFERNAN, A.E.; BISKUP, J.J.; KRAMEK, B.A.; ANDERSON, G.M. Simultaneous staphylectomy and unilateral arytenoid lateralization in dogs presenting for dyspnea: 23 cases (2010-2013). Canadian Veterinary Journal, v.57, n.10, p. 1087-1093, 2016. Disponível em: < https://www.ncbi.nlm.nih.gov/pmc/articles/PMC5026151/>

HEIDENREICH, D.; GRADNER, G.; KNEISSL, S.; DUPRE, G. Nasopharyngeal Dimensions from Computed Tomography of Pugs and French Bulldogs with Brachycephalic Airway Syndrome. Veterinary Surgery, v.45, n.1, p. 83-90, 2016. Disponível em: <https://www.ncbi.nlm.nih.gov/pubmed/26731598> doi: 10.1111/vsu.12418

HOSTNIK, E.T.; SCANSEN, B.A.; ZIELINSK, R.; GHADIALI, S.N. Quantification of nasal airflow resistance in English bulldogs using computed tomography and computational fluid dynamics. Veterinary radiology \& ultrasound: the official journal of the American College of Veterinary Radiology and the International Veterinary Radiology Association, v. 58, n.5, p.542-551, 2017. Disponível em: <https://www.ncbi.nlm.nih.gov/pubmed/28718208> doi: 10.1111/vru.12531.

HUGHES, J.R.; KAYE, B.M.; BESWICK, A.R.; TER HAAR, G. Complications following laryngeal sacculectomy in brachycephalic dogs. Journal Small Animal Practice, v.59, n.1, p. 16-21, 2018. Disponível em: <https://www.ncbi.nlm.nih.gov/pubmed/29047114>. doi: 10.1111/jsap.12763.

KIRSCH, M.S.; SPECTOR, D.; KALAFUT, R.S.; MOORE, G.S.; MC DOUGALL R. Comparison of carbon dioxide laser versus bipolar vessel device for staphylectomy for the treatment of brachycephalic obstructive airway syndrome. Canadian Veterinary Journal, v.60, n.2, p.160-166, 2019.

$\mathrm{KOCH}$, D.A.; ARNOLD, S. HUBLER, M.; MONTAVON, P.M. Brachycephalic syndrome in dogs. Compendium: Continuing education for veterinarians, v.25, n.1, p.48-55, 2003. Disponível em: https://dkoch.ch/fileadmin/user upload/Publikationsliste/Weichteile/Koch et al Brac hycephalic syndrome Compendium 2003.pdf>

LEONARD, H.C. Collapse of the larynx and adjacent structures in the dog. Journal American Veterinary Medicine Association, v.137, n.1, p.360-363, 1960. Disponível em: < https://www.ncbi.nlm.nih.gov/pubmed/14415784>

LIU, N.C.; GENAIM, M.A.; KALMAR, R.; SARGAN, D.R.; LADLOW, J.F. Objective effectiveness of and indications for laser-assisted turbinectomy in brachycephalic obstructive airway syndrome, Veterinary Surgery, v.48, n.1, p.79-87, 2018. 
Disponível em: < https://www.ncbi.nlm.nih.gov/pubmed/30303538 > doi: $10.1111 /$ vsu. 13107

LIU, N.C.; OECHTERING, G.U.; ADAMS, V.J.; KALMAR, R.; SARGAN, D.R et al. Outcomes and prognostic factors of surgical treatments for brachycephalic obstructive airway syndrome in 3 breeds. Veterinary Surgery, v.46, n.2, p. 271-280, 2017b. Disponível em: < https://www.ncbi.nlm.nih.gov/pubmed/28146288> doi: 10.1111/vsu.12608

LIU, NC.; TROCONIS, E.L.; KAMAR, L.; PROCE, D.J.; WRIGHT, H.E.; et al. Conformational risk factors of brachycephalicobstructive airway syndrome (BOAS) in pugs, French bulldogs, and bulldogs. Plos One, v.12, n.8, p.1-24, 2017a. Disponível em:<https://doi.org/10.1371/journal.pone.0181928> oi:10.1371/journal.pone.0181928

LODATO, D.L.; HEDLUND, C.S. Brachycephalic airway syndrome: pathophysiology and diagnosis. Compendium: Continuing education for veterinarians, v.34, n.7, p.3-5, 2012. Disponível em: < https://www.ncbi.nlm.nih.gov/pubmed/22847322>

LODATO, D.; MAUTERER, J. Techniques for performing corrective surgery: dogs with brachycephalic airways syndrome. Today's veterinary practice, v.4, n.1, p.7883, 2014. Disponível em: <https://todaysveterinarypractice.com/wpcontent/uploads/sites/4/2016/06/T1401C09.pdf>

MATHEWS, K.G. Correction of overlong soft palate. In: LOMMER, M.J.; VERSTRAET, F.J.M. Oral and Maxillofacial Surgery in Dogs and Cats. California: Ed Saunders, 2012. Cap 56, p.539-542. Disponível em: $<$ http://doi.org/10.1016/C2010-0-68695-0>

MAZZARO, L.; LEON-ROMAN, M. A. Correção de estenose de narina - Relato de caso. Revista de Educação Continuada em Medicina Veterinária e Zootecnia do CRMV-SP, v.14, n.2, p.55-55, 2016. Disponível em: <https://www.revistamvezcrmvsp.com.br/index.php/recmvz/article/view/31887>

MILLER, J.; GANNON, K. Perioperative Management of Brachycephalic Dogs. Clinician's Brief, v.13, n.4, p.54-59, 2015. Disponível em: $<$ https://www.cliniciansbrief.com/article/perioperative-management-brachycephalicdogs $>$.

OECHTERING, G. Brachycephalic syndrome - new information on an old congenital disease. Veterinary Focus, v.20, n.2, p. 2-9, 2010. Disponível em: https://www.researchgate.net/publication/285703902_Brachycephalic_syndromenew_information_on_an_old_congenital_disease>

OECHTERING, G.U.; POHL, S.; SCHULUETER, C.; LIPPERT, J.P.; ALEF, M. et al. A novel approach to brachycephalic syndrome. 1. Evaluation of anatomical intranasal airway obstruction. Veterinary Surgery, v.45, n.1, p.165-172, 2016. Disponível em: $<$ https://www.ncbi.nlm.nih.gov/pubmed/26790550> doi 10.1111/vsu.12446 
OECHTERING, T.H.; OECHTERING, G.U.; NOLLER, C. Structural characteristics of the nose in brachycephalic dog breeds analysed by computed tomography. Tierarztliche Praxis, v. 35, n.3, p.177-187, 2007. Disponível em: < https://www.researchgate.net/publication/285682745_Structural_characteristics_of_t he_nose_in_brachycephalic_dog_breeds_analysed_by_computed_tomography>

PACKER, R.M.A.; HENDRICKS, A.; TIVERS, M.S.; BURN, C.C. Impact of facial conformation on canine health: Brachycephalic obstructive airway síndrome. Plos One, v.10, n.10, p.1-21, 2015. Disponível em: < https://doi.org/10.1371/journal.pone.0137496> doi: 10.1371/journal.pone.0137496

PACKER, R.M.A.; TIVERS, M.S. Strategies for the management and prevention of conformation-related respiratory disorders in brachycephalic dogs. Veterinary Medicine: Research and Reports, v.6, n.1, p. 219-232, 2015. Disponível em:< https://www.ncbi.nlm.nih.gov/pmc/articles/PMC6067768/> 10.2147/VMRR.S60475

PHILLIPS, H.; JAEGER, M. Brachycephalic Syndrome. Veterinary Team Brief, v.14, n.9, p.31-37, 2016. Disponível em: https://www.cliniciansbrief.com/article/brachycephalic-syndrome

PICHETTO, M.; ARRIGUI, S.; ROCCABIANCA, P.; ROMUSSI, S. The anatomy of the dog soft palate. II. Histological Evaluation of the caudal soft palate in brachycephalic breeds with grade I brachycephalic airway obstructive syndrome. The anatomical record, v.298, n.3, p.618-623, 2015. Disponível em: $<$ https://www.ncbi.nlm.nih.gov/pubmed/21634020> doi: 10.1002/ar.21417

PINK, J.J.; DOYLE, R.S.; HUGHES, J.M.; TOBIN, E.; BELLENGER, C.R. Laryngeal collapse in seven brachycephalic puppies. Journal Small Animal Pratice, v.47, n.3, p.131-135, 2006. Disponível em: <https://www.ncbi.nlm.nih.gov/pubmed/16512844> doi: $\underline{10.1111 / j .1748-5827.2006 .00056 . x}$

RIECKS, T.W.; BIRCHARD, S.J.; STEPHENS, A.J. Surgical correction of brachycephalic syndrome in dogs: 62 cases (1991-2004). Journal of the American Veterinary Medical Association, v.230, n.9, p.1324-1328, 2007. Disponível em: <https://doi.org/10.2460/javma.230.9.1324>

SCHUENEMANN, R.; OECHTERING, G.U. Inside the brachycephalic nose: intranasal mucosal contact points. Journal of the American Animal Hospital Association, v. 50, n.1, p.149-158, 2014. Disponível em: < https://www.ncbi.nlm.nih.gov/pubmed/24659729> doi: 10.5326/JAAHA-MS-5991

SCHUENEMANN, R.; POHL, S.; OECHTERING, G.U. A novel approach to brachycephalic syndrome. 3 . Isolated laser-assisted turbinectomy of caudalaberrant turbinates (CAT LATE). Veterinary Surgery, v.46, n.1, p.32-38, 2016. Disponível em: < https://www.ncbi.nlm.nih.gov/pubmed/28052420> doi: 10.1111/vsu.12587.

SOUTO, C.K.; MARTÍN, C.M.; FERRANTE, B.; PINTO, A.C.C.F. Métodos de diagnóstico por imagem para avaliação traqueal em pequenos animais-Imaging 
methods for tracheal assessment in small animals. Revista Acadêmica Ciência Animal, v.13, n.1, p. 11-123, 2015. Disponível em: http://dx.doi.org/10.7213/academica.13.FC.AO12.

TORREZ, C.V.; HUNT, G.B. Results of surgical correction of abnormalities associated with brachycephalic airway obstructive syndrome in dogs in Australia. Journal of Small Animal Practice, v.47, n.3, p. 150-154, 2006. Disponível em: https://www.ncbi.nlm.nih.gov/pubmed/16512847doi10.1111/j.1748-827.2006.00059.x

TRAPPLER, M.; MOORE, K. Canine brachycephalic airway syndrome. Vet learns Compendium, v.33, n.3, p1-7, 2011. Disponível em: https://www.ncbi.nlm.nih.gov/pubmed/21870354

TROSTEL, C.D.; FRANKEL, J.D. Punch Resection Alaplasty Technique in Dogs and Cats With Stenotic Nares: 14 Cases. Journal of the American Animal Hospital Association, v.46, n.1, p. 5-11, 2010. Disponível em: < https://doi.org/10.5326/0460005>

WHITE RN. Surgical management of laryngeal collapse associated with brachycephalic airway obstruction syndrome in dogs. Journal of Small Animal Practice, v.53, n.1, p.44-50, 2012. Disponível em: < https://doi.org/10.1111/j.17485827.2011.01156.x 